\title{
3 Einleitung
}

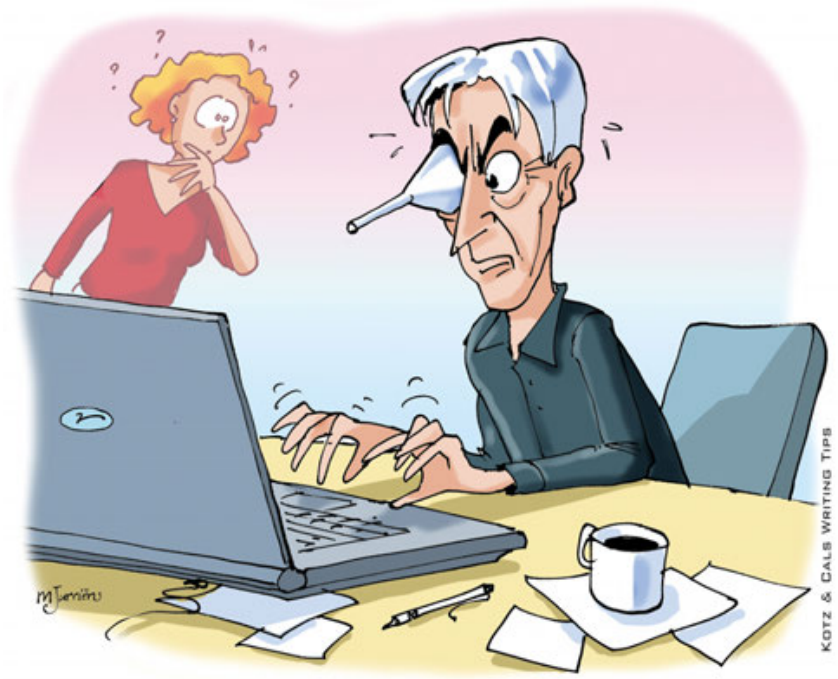

Tipp 3 - Einleitung: arbeite an der Trichterform!

\section{Was Sie wissen sollten}

Heutzutage bevorzugen viele Redakteure und Gutachter von empirischen Artikeln kurze und gezielte Einleitungen. Der Zweck der Einleitung ist es, den Lesern anhand wesentlicher Informationen zu verdeutlichen, warum Sie die Studie durchgeführt haben, und die Fragestellung der Studie zu benennen. Sie legt den Kontext der vorgestellten Studie fest, indem sie die bisherige relevante Literatur (mit Verweisen) sowie die derzeitigen Ansichten zum von Ihnen untersuchten Problem zusammenfasst. Die Einleitung muss Lesern ermöglichen, die biologischen, klinischen oder methodischen Gründe für Ihre Studie zu verstehen. Sie sollte auf die Zeitschrift zugeschnitten sein, bei der Sie den Artikel einreichen wollen. Eine gute Einleitung wird die Studie an Redakteure, Gutachter, Leser und manchmal sogar an die Medien „verkaufen“.

Die Struktur einer Einleitung kann als Trichter gesehen werden. Der breiteste Teil oben, am Anfang, repräsentiert den allgemeinen Kontext des Studienthemas. Danach verengt er sich zu spezifischen Kontextinformationen und mündet in der Begründung der Studie (anhand der Problemstellung) sowie - unerläss- 
lich - der Fragestellung oder Zielsetzung der Studie. Die Einleitung hat keine maximale Anzahl von Wörter wie die Zusammenfassung (Abstract), sollte jedoch so präzise wie möglich gehalten werden und typischerweise nicht mehr als $10-15 \%$ der Gesamtwortzahl des Artikels umfassen. Die Einleitung ist der Beginn des Erzählstrangs Ihres Artikels - fangen Sie also erst mit dem Schreiben an, wenn Sie eine Gesamtstruktur Ihres Artikels vor Augen haben.

\section{Was Sie tun sollten}

Fragen Sie sich, ob Sie mit der Gesamtstruktur zufrieden sind. Werfen Sie einen Blick auf die Gliederung Ihrer Einleitung und wählen Sie die wichtigsten Leitsätze (siehe Teil 1: Anfangen zu Schreiben). Nehmen Sie diese Leitsätze und entwickeln Sie daraus vier bis fünf Absätze, wobei Sie das Trichtermodell im Hinterkopf haben. Denken Sie an die Relevanz Ihrer Studie, die Diskussion bestehender Evidenz, eventuelle Lücken in der Evidenz und das Ziel (Versprechen) des aktuellen Artikels. Obwohl viele Wissenschaftler Zeitschriften als statische Publikationsquellen sehen, dienen sie einem höheren Zweck: der Förderung wissenschaftlicher Debatte und Kommunikation.

Die Einleitung soll keine vollständige Literaturübersicht des gesamten Feldes sein, das Sie erforschen. Sie sollte Lesern das Verständnis ermöglichen, warum Sie diese Studie in Angriff genommen haben und warum die spezifischen Ziele von Bedeutung sind. Diskutieren Sie zuerst den allgemeinen Hintergrund Ihrer Studie, vorzugsweise unter Betonung der Größenordnung (Prävalenz/Inzidenz) oder der gesellschaftlichen Last, die durch die Krankheit oder das Problem entsteht. Zeigen Sie dann auf, was zu dem Thema bereits bekannt und was noch unbekannt ist. Hier sollten Sie einen Bezug zur späteren Diskussion des Artikels herstellen, zu viele Überschneidungen jedoch vermeiden. Belassen Sie detaillierte Vergleiche mit anderen Studien für die Diskussion. Zeigen Sie Lücken in der Evidenz auf und erklären Sie, warum die Erkenntnisse Ihrer Studie relevant ist.

Zögern Sie nicht hervorzuheben, warum diese Studie nötig und wichtig ist. Berücksichtigen Sie, was Leser von Ihrer Studie lernen könnten, und heben Sie dies hervor. Gehen Sie dann über zur Problemstellung des Artikels; dies ist der eigentliche Beginn Ihres Erzählstrangs. Denken Sie daran, dass der letzte Absatz der Einleitung die Aufmerksamkeit der Leser erregen sollte. Beenden Sie demzufolge die Einleitung mit Ihrer Fragestellung und eventuell Ihrer Hypothese und erklären Sie kurz, was Sie getan haben, um diese Frage zu beantworten. Versuchen Sie, dies damit zu kombinieren, was zur Beantwortung der Frage unternommen wurde, vorzugsweise unter Angabe des Studiendesigns. Dies schafft 
eine schöne Brücke zum Abschnitt Methoden, in dem Sie Ihre Herangehensweise detailliert erklären. Trennen Sie klar die größeren (primären) von den kleineren (sekundären) Fragestellungen. Seien Sie zurückhaltend beim Benennen von sekundären Fragestellungen, aber wenn Sie diese erwähnen wollen, benutzen Sie einen getrennten Satz und stellen Sie sicher, dass diese als sekundär gekennzeichnet werden.

Benutzen Sie eine klare, saubere und emotionslose Sprache. Versuchen Sie, überwiegend aktive Verben zu verwenden, und erwägen Sie die Benutzung von Signalwörtern (z. B. „um festzustellen ob“, „zu klären“, „zu vergleichen“). Benutzen Sie die Gegenwartsform für allgemein anerkannte Tatsachen (z. B. „Kreuzschmerzen sind ein häufiger Grund, um Physiotherapeuten aufzusuchen“) und die Vergangenheitsform für spezifische Erkenntnisse, die noch nicht als allgemein anerkannt gelten (z. B. „zwei Behandlungen pro Woche zeigten in einer Kohortenstudie ein besseres Ergebnis als eine Behandlung“). Untermauern Sie wichtige Aussagen mit Literaturhinweisen und zitieren Sie dabei die Quelle der Originaldaten. Wählen Sie nur die wirklich relevanten Literaturhinweise und suchen Sie die wichtigsten aus, wenn Sie mehrere Optionen haben. Beachten Sie, dass Redakteure Verweise auf Artikel in deren eigener Zeitschrift schätzen, da Sie damit zeigen, ein Interesse an den Inhalten zu haben und ein aktiver Teil der wissenschaftlichen Debatte der Zeitschrift zu sein, und weil hiermit möglicherweise Zitationsraten gefördert werden.

\section{Checkliste für die Einleitung}

- Prüfen Sie, ob die Einleitung eine Trichterform mit klaren Absätzen hat bezüglich:

- dem allgemeinen Hintergrund (worum geht es hier?),

- was bereits bekannt und was noch unbekannt ist (warum war diese Studie notwendig und wichtig?),

- der primären Fragestellung (was wollten wir wissen?) und

- dem Studienziel und -design (was haben wir getan, um die Fragestellung zu beantworten?)

- Begrenzen Sie die Länge der Einleitung (maximal 10-15\% der Gesamtwortzahl)

- Überprüfen Sie, ob die Einleitung den Beginn Ihres Erzählstrangs gut markiert, indem Sie sich die Gesamtstruktur Ihres Artikels ansehen

- Fragen Sie sich: „Kann ich mit dieser Einleitung meinen Artikel an Redakteure, Gutachter, Leser und die Medien gut verkaufen?“ 